\title{
Regulation of epidermal growth factor receptor signaling by plasmid-based MicroRNA-7 inhibits human malignant gliomas growth and metastasis in vivo
}

W. WANG ${ }^{1, \ddagger}$, L. X. DAI ${ }^{1, \ddagger}$, S. ZHANG ${ }^{2}$, Y. YANG ${ }^{1}$, N. YAN ${ }^{1}$, P. FAN ${ }^{1}$, L. DAI ${ }^{1}$, H. W. TIAN ${ }^{1}$, L. CHENG ${ }^{1}$ X. M. ZHANG ${ }^{1}$, C. LI ${ }^{1}$, J. F. ZHANG ${ }^{1}$, F. XU' ${ }^{1}$, G. SHI ${ }^{1}$, X. L. $\mathrm{CHEN}^{1}$, T. DU ${ }^{1}$, Y. M. $\mathrm{LI}^{1}$, Y. Q. WEI ${ }^{1}, \mathrm{H}$. X. DENG ${ }^{1, *}$

${ }^{1}$ State Key Laboratory of Biotherapy, West China Hospital, Sichuan University, Chengdu, Sichuan, the People's Republic of China; ${ }^{2}$ West China Medical School, Sichuan University, West China Hospital, Chengdu, Sichuan, The People's Republic of China;

${ }^{*}$ Correspondence: denghongx@scu.edu.cn

${ }^{*}$ Contributed equally to this work.

Received March 16, 2012 / Accepted November 29, 2012

\begin{abstract}
MicroRNAs are endogenous, non-coding RNAs of approximately 20-22 nucleotides that regulate genes expression by binding to the 3' untranslated region (UTR) of targets mRNAs and play critical roles in cancer pathways. Malignant glioma is the most common and highly lethal central nervous system tumor for which little effective treatment is available over several decades. The purpose of this study was to explore the therapeutic potential of plasmid-based microRNA-7 (miR-7) for gliomas in vivo. Enhancing miR-7 levels in vitro could significantly induce cell apoptosis, and inhibit cell proliferation, cell migration and invasion. Western blotting analysis was performed, which indicated that miR-7 directly inhibited epidermal growth factor receptor (EGFR) and further antagonized the downstream protein kinases including ERK, Akt and Stat3. Furthermore, systemic administration of miR-7 encapsulated in cationic liposome resulted in glioma xenografts growth arrest and the metastatic nodules decrease effectively in a sequence-specific manner. In this study, miR-7 was applied in glioma treatment for the first time in vivo. Our findings suggested that the plasmid-mediated gene therapy with miR-7 appeared to be a promising candidate for the development of new antitumor and anti-metastasis treatment for human glioma.
\end{abstract}

Key words: miR-7, glioma, metastasis, apoptosis, gene therapy

Malignant glioma is the most common and lethal primary brain tumors in adults. The deadly nature of malignant gliomas resides in their explosive cell proliferation, intense resistance to cell apoptosis and widespread infiltration throughout the brain. Despite multimodal therapies such as surgery, radiotherapy and chemotherapy, the median survival of glioblastoma (GBM) is less than 1 year [1]. Novel therapeutic approaches are needed to improve long-term survival for this cancer. Recent advances in our understanding of the altered genes and pathways in malignant gliomas offer the opportunities for new therapeutic strategy based on targeting essential molecular mechanisms.

MicroRNAs (miRNAs) are small, non-protein-coding, single-stranded RNAs that regulate target genes expression post-transcriptionally in normal tissues and cancers. miRNAs bind to the 3' UTR of target gene and lead repression or degradation of the transcript through imperfect or complete complementarity. The partial complementarity allows miRNAs to target 3' UTR of multiple genes [2-4], and growing evidences indicate that miRNAs are involved in a range of processes including cellular development, apoptosis and disease in human [5-8]. A recent study demonstrates that more than $50 \%$ of miRNA genes are present in cancer associated genomic regions or fragile sites [9], suggesting that miRNAs may play important roles in cancer pathogenesis, diagnosis and progression [10-12].

The EGFR, a member of the erbB receptor family which widely express in all tissues and regulate the normal cellular processes, is frequently amplified in a variety of human malignancies, especially glioblastoma multiforme [13-16]. In GBM patients, EGFR amplification was a significant predictor for poor survival prognosis [17], thus EGFR and its downstream members are ideal therapeutic targets. 
MiR-7 is an intronic miRNA that resides in the first intron of heterogeneous ribonucleoprotein $\mathrm{K}$ gene on chromosome 9 and conserved across all species. In previous reports, it has been reported that IGF1R (insulin-like growth factor 1 receptor) is targeted by miR-7 in tongue squamous cell carcinoma cells which in turn resulted in a reduction of cell proliferation and an enhanced apoptotic rate. And PAK1, a serine/threonine kinase that plays a pivotal role in cell migration and invasion, is also targeted by miR-7. The recent findings show the relationships between miR-7 and the proto-oncogenes (e.g. PAK1, RAF1 and EGFR) suggest that miR-7 plays major roles in tumorigenesis [18-21].

It is well known that the mature miR-7 expression dramatically decreased in glioma compared with adjacent normal brain tissues [21, 22], Excitingly, the EGFR expression was negatively regulated by miR-7 [21], and deficiency of miR-7 function in glioma cells cause downstream molecules switch on or off, which in turn affects glioma cells vitality and disease process.

To date, most researches have been done to explore the functional roles for miR-7 in various cells in vitro, few are but focused on its treatment value in vivo. In our current study, we firstly presented the demonstration that systemic delivery of plasmid-mediated miR-7 encapsulated in cationic liposome significantly inhibited glioma cell growth and metastasis in vivo. These findings suggested that miR-7 should be a potential approach for human malignant glioma therapy by targeting EGFR signaling.

\section{Materials and methods}

Plasmid construction. A 150-base-pair genomic fragment spanning the hsa-miR-7 was inserted into pGenesil-2.1 expression vector (Genesil Biotechnology Company, Wuhan, China) at its BamHI / HindIII sites to express miR-7. All the sequences were confirmed by DNA sequencing. The empty expression plasmid named $\mathrm{KB}$ was designated as control. Plasmids were prepared using Endo-free Plasmid Giga kit (Qiagen, Germantown, MD, USA) according to the manufacturer's direction.

Cell culture and transfection. Human glioma cell lines U-87MG and U-118MG were acquired from the American Type Culture Collection (ATCC, Rockville, MD, USA), and U251 was obtained from Institute of Biochemistry and Cell Biology, Chinese Academy of Sciences. Cells were cultured in Dulbecco's modified Eagle's medium (DMEM) supplemented with $10 \% \mathrm{FBS}$ at $37^{\circ} \mathrm{C}$ under $5 \% \mathrm{CO}_{2}$ in atmosphere. Plasmids miR-7 and KB ( $2 \mu \mathrm{g}$ each) were transfected into cells seeded in six-well plates by Fugene HD Transfection Reagent (Roche Applied Science, Indianapolis, IN, USA) following the manufacturer's instruction.

In vitro proliferation analysis. Cell proliferation was analyzed by colony formation assay and cell cycle analysis. For colony formation assay, cells transfected with miR-7, KB or untreated were trypsinized and seeded at 500 cells/well in six-well plates. For 8-11 days later, cells were stained with crys- tal violet and aggregation of more than 50 cells was regarded as colony. For cell cycle analysis, cells were synchronised for $24 \mathrm{~h}$ by thymine (Sigma, St Louis, MO, USA) and then treated with blank, KB or miR-7. 48h later, cells were harvested and cell cycle analysis was done by FCM using propidium iodide (PI) staining method.

In vitro apoptosis analysis. Cell apoptosis in vitro was analyzed by flow cytometric analysis and Hoechst 33258 staining. $48 \mathrm{~h}$ after transfection, for flow cytometric analysis, cells were collected and suspended in $1 \mathrm{~mL}$ PI/RNase/PBS $(100 \mu \mathrm{g} / \mathrm{ml}$ propidium iodide and $10 \mu \mathrm{g} / \mathrm{ml}$ RNase A). The DNA content was analyzed by flow cytometry (Beckman Coulter, Fullerton, CA, USA) and cells stayed in sub-G1 phase were regarded as apoptotic cells. For Hoechst 33258 staining, cells were stained with Hoechst 33258 (Sigma, St Louis, MO, USA) staining solution as instructed by the manufacturer and analyzed by fluorescence microscopy.

Cell migration analysis (Wound healing analysis). $24 \mathrm{~h}$ after transfection, monolayer cells which were grown to 70$80 \%$ confluence in six-well plates were scratched by a plastic pipette and cultured with fresh DMEM medium containing $5 \%$ FBS. Images were taken by an inverted microscope for $0 \mathrm{~h}$ and $24 \mathrm{~h}$, and the percentage of inhibition was expressed using untreated cells as $100 \%$.

Cell invasion analysis. The transwell filters (Millipore, Billerica, MA, USA) were coated with Matrigel (BD Biosciences,

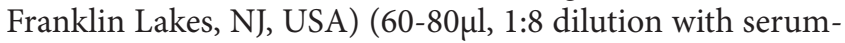
free DMEM) and incubated at $37^{\circ} \mathrm{C}$ for $1 \mathrm{~h}$. When matrigel became solidified, the top chambers were seeded with $200 \mu \mathrm{l}$ serum-free DMEM and transfected tumor cells $\left(2 \times 10^{5}\right.$ per well), the bottom chambers were filled with DMEM medium containing $20 \% \mathrm{FBS}$ and various growth factors. $24 \mathrm{~h}$ later, non-invaded cells on the upper side of the membrane were scraped off with a cotton swab and migrated cells were stained with $0.05 \%$ crystal violet. The invaded cells were counted and photographed under a light microscope.

Western blotting analysis. Cells transfected for $48 \mathrm{~h}$ were lysed on ice for $30 \mathrm{~min}$ by RIPA Lysis Buffer (Beyotime Biotechnology, China). For tissue samples, the frozen tissue was powdered in liquid nitrogen and lysed in RIPA lysis buffer. Equal doses protein from each sample were applied to SDS-PAGE gels and probed with specific antibodies including EGFR, MMP-2, MMP-9, Survivin, PCNA, phospho-Stat3 (Ser727), Stat3, phospho-ERK1/2 (Thr202/Tyr204), ERK1/2, phospho-Akt (Ser473), Akt (Cell Signaling Technology, Boston, MA, USA) and GAPDH (Sigma, St Louis, MO, USA).

Cationic liposome and liposome-DNA complex preparation. Cationic liposome for treatment in vivo was prepared by using DOTAP (Alabaster, AL, USA): cholesterol (SigmaAldrich, St. Louis, MO) at 1: 1 molar ratio hydrated in 5\% dextrose solution and sequentially extruded through a $100 \mathrm{~nm}$ polycarbonate filter [23]. For in vivo administration, liposomes and plasmids were diluted in 5\% glucose and gently mixed with a liposome/ DNA weight ratio of 5:1 (5 $\mu \mathrm{g}$ plasmid/25 $\mu \mathrm{g}$ 


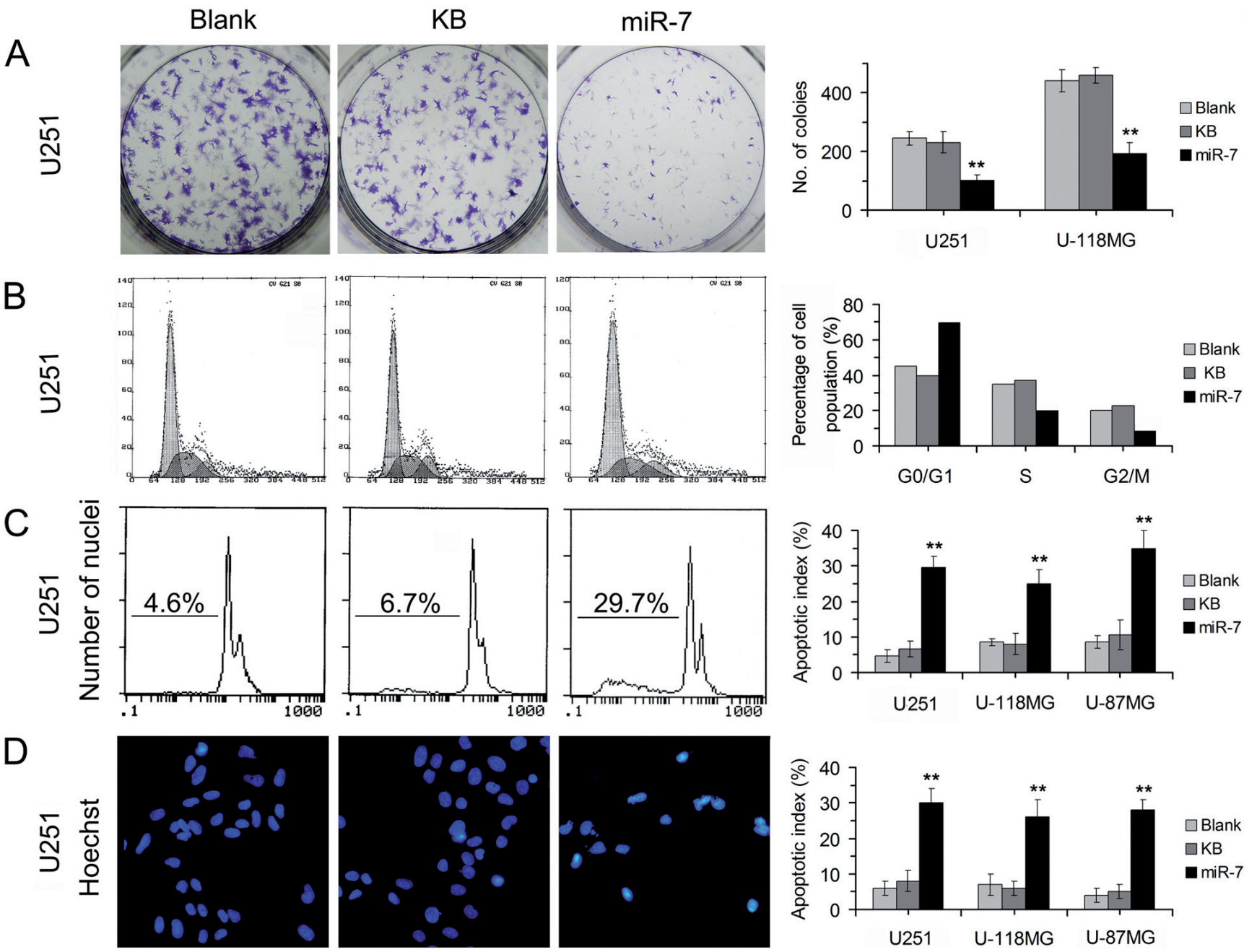

Figure 1. Transfection with miR-7 inhibited glioma cell proliferation and induced cell apoptosis. 48h after transfection with miR-7, KB or untreated, colony formation assay in U251 and U-118MG cells (A), cell cycle analysis in U251 cells (B), quantitative assessment of apoptotic cells by flow cytometric in U251, U-118MG and U-87MG cells $(C)$, and morphological changes examination by Hoechst 33258 staining (original magnification, $\times 200)$ in U251, U-118MG and U-87MG cells (D) were performed. Columns, mean; bars, SD (n=3; ANOVA; ${ }^{\star *}, P<0.01$ versus controls).

liposome/100 $\mu$ l total volume per mouse). The resulting complexes were incubated at room temperature for $30 \mathrm{~min}$ before intravenous injection in vivo.

Animal experiments. All animal protocols were approved by the Institutional Animal Care and Treatment Committee of Sichuan University (Chengdu, Sichuan, China). U-87MG $\left(5 \times 10^{6}\right.$ cells $)$ and U251 $\left(3 \times 10^{6}\right.$ cells $)$ in $0.1 \mathrm{ml}$ serum-free DMEM medium were inoculated subcutaneous into the right flank of each female athymic mouse (3-4 weeks old), respectively. When the size of tumor reached around 100 $\mathrm{mm}^{3}$, the animals were randomly divided into three groups (5 mice per group): 5\% GS, KB and miR-7. Tumor-bearing mice were treated intravenously through the tail vein every two days for 4 weeks (U-87MG) or 5 weeks (U251), and the tumor volume was measured with a caliper every 3 days and calculated according to the formula: Tumor volume = length $\times$ width $2 / 2$. Animals were sacrificed three days after the last injection, and solid tumor tissues were removed and weighed. Part of the tumor tissues was fixed in $4 \%$ paraformaldehyde and embedded in paraffin, and the rest was immediately frozen.

Since U251 xenograft model can spontaneous formed pulmonary and lymph node metastasis after 4-5 weeks inoculation, lungs and lymph node tissues of the animals were excised. Tumor metastases on lung surfaces were counted under a dissecting microscope and photographed after be fixed in $4 \%$ paraformaldehyde solution, subsequently the lung and lymph node tissues were paraffin embedded and sectioned for further pathologic analysis.

Detection of apoptosis. Cell apoptosis in situ was determined using TUNEL assay kit (Promega, San Luis Obispo, CA, USA) according to the manufacturer's protocol. The number 

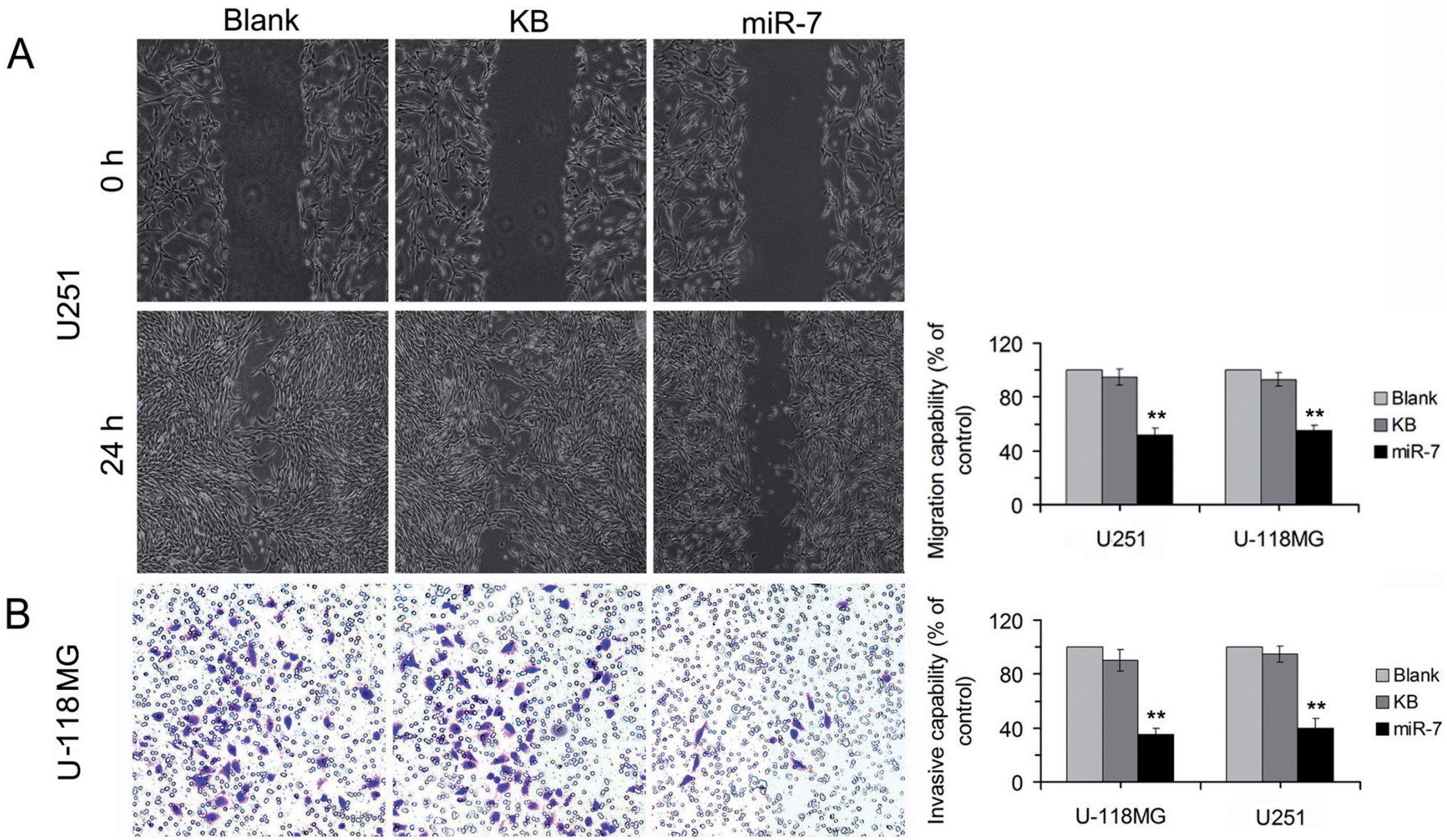

Figure 2. Transfection with miR-7 inhibited glioma cell migration and invasion. A, photomicrographs of wound healing analysis for $0 \mathrm{~h}$ and $24 \mathrm{~h}$ with migrating U251 cells are shown. Plots showed the percentage of cell migration inhibition in U251 and U-118MG glioma cell lines treated with miR-7, KB or untreated $\left(\mathrm{n}=3\right.$; ANOVA; ${ }^{* *}, P<0.01$ versus controls). B. photomicrographs of a sample transwell membrane with invading $\mathrm{U}-118 \mathrm{MG}$ cells are shown. Plots showing mean number of invading cells in U-118MG and U251 glioma cell lines treated with miR-7, KB or untreated ( $n=3$; ANOVA; ${ }^{* *}$ $P<\mathbf{0 . 0 1}$ versus controls).

of TUNEL-positive cells which represented apoptotic cells were counted under a fluorescence microscopy $(\times 200)$ in six randomly selected fields. The apoptotic index was defined as follows: apoptotic index $(\%)=100 \times$ apoptotic cells/total tumor cells.

Quantitative real time PCR. As described previously [24], total RNA was extracted from glioma cancer cells and xenograft tumors using Trizol reagent (Invitrogen, Carlsbad, CA, USA), and quantitative real time PCR (qRT-PCR) analysis was performed to detect the expression of candidate miRNAs with the TaqMan MicroRNA assays kit (Applied Biosystems, Foster City, CA, USA).

Statistical analysis. All statistical analyses were performed using SPSS 13.0. Data were expressed as the mean \pm SD and were analyzed statistically using one-way ANOVA. Differences were considered statistically significant if $P<0.05$.

\section{Results}

Effect of miR-7 on human glioma cell lines. To detect the functional effects of miR-7 on human glioma cell viability in vitro, cell proliferation analysis, flow cytometric analysis, cell migration and invasion analysis was performed. According to the results of sequent experiments, a decrease in the number of cell colonies, an increased percentage of cells in G0 / G1 phase (Fig. 1A, 1B), an elevation of sub-G1 phase cell population (Fig. 1C), a reduced area of cell migration and depressed cell invasion ability (Fig. 2A, 2B) were distinctly observed in group treated with miR-7. Hoechst 33258 staining was also performed to assess cell apoptosis by morphological changes. More condensed nucleus which were characteristic of apoptosis, were captured by fluorescence microscope in miR-7 treated group (Fig. 1D), whereas there were no significant changes in control groups. These results indicated that glioma cells transfected with miR-7 in vitro could evidently inhibit cell growth, cell migration, invasion, meanwhile induce cell apoptosis.

Regulation of miR-7 on EGFR signaling pathways. EGFR overexpression leads to the activation of various downstream signaling effectors that are responsible for important cellular processes, such as proliferation, apoptosis, cell migration and invasion. To investigate whether miR-7 inhibited EGFR and its downstream signaling members, we screened some essential kinases involved in EGFR signaling pathway, and the results were consistent with the effect discussed above. MiR-7 significantly suppressed the phosphorylation of Akt, 


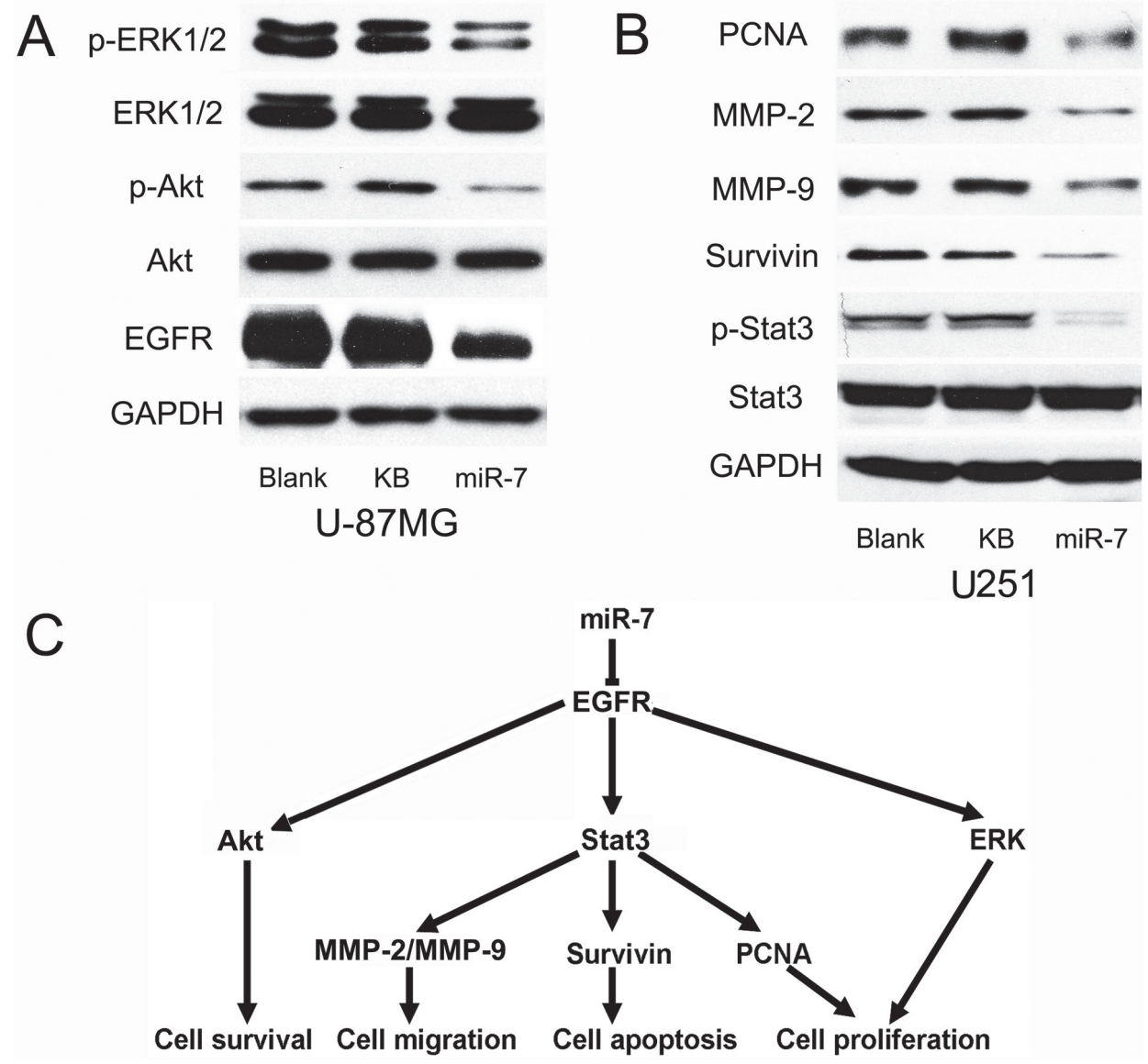

Figure 3. miR-7 inhibited EGFR activity and its downstream signaling effectors. A, immunoblots of phospho-ERK1/2 (Thr202/Tyr204), ERK1/2, phospho-Akt (Ser473), Akt, EGFR proteins in U-87MG cells 48h after transfection with miR-7, KB or untreated. GAPDH serves as loading controls. B, immunoblots of MMP-2, MMP-9, Survivin, PCNA, phospho-Stat3 (Ser727) and Stat3 proteins in U251 cells 48h after transfection with miR-7, KB or untreated. GAPDH serves as loading controls. C, diagram of signaling pathway for miR-7 regulate cell vitality.

ERK and Stat3, as well as MMP-2, MMP-9, Survivin and PCNA (Fig. 3A, 3B), which suggested that miR-7 exerted its antitumor function by direct targeting EGFR on the surface of glioma cells and further antagonizing EGFR-mediated downstream signaling cascade (Fig. 3C).

Effect of miR-7 on tumor progression in vivo. To further investigate the effects of miR-7 on tumor progression in vivo, subcutaneous xenografts of glioma were established and treated as described in the Materials and Methods. Compared with control groups, primary tumor growth were sharply suppressed and mean tumor weight were evidently reduced in miR-7 group (Fig. 4A, 4B; $P<0.01$ ); conversely, no significant differences were observed between the two control groups $(P>0.05)$. Moreover, to explore the effects of miR-7 on cells apoptosis in vivo, TUNEL analysis was carried out in tumor sections. In coincidence with the results in vitro, TUNEL-positive nuclei (with green staining) were increased significantly in mice treated with miR-7 (Fig. 4C, $P<0.01)$.
Furthermore, to verify the antitumor effects caused by miR-7 treatment, we detected the mature miR-7 in glioma subcutaneous xenografts by qRT-PCR. Compared with control groups, the expression of miR-7 sharply increased in group treated with miR-7 (Fig. 5B, $P<0.05$ ), whereas there were no significant differences between the two control groups $(P>$ 0.05). And the results were consistent with the detection in vitro (Fig. 5A). Meanwhile, the EGFR and PCNA expression in tumors treated with miR-7 were obviously down-regulated (Fig. 5C, D).

In addition, to evaluate potential drug toxicity on mice during the treatment, we continuously evaluated relevant indexes such as weight, appetite, diarrhea and behavior. No significant side reactions were found in gross measures. Moreover, no pathological changes in heart, liver, spleen, lungs, or kidneys of treated mice were detected by microscopic examination (data not shown).

Effect of miR-7 on U251 lung and lymph node metastases. Untreatable metastasis was the main cause of mortality in pa- 

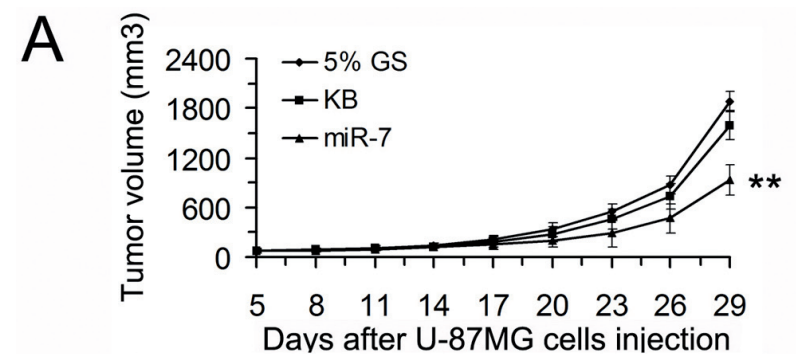

B
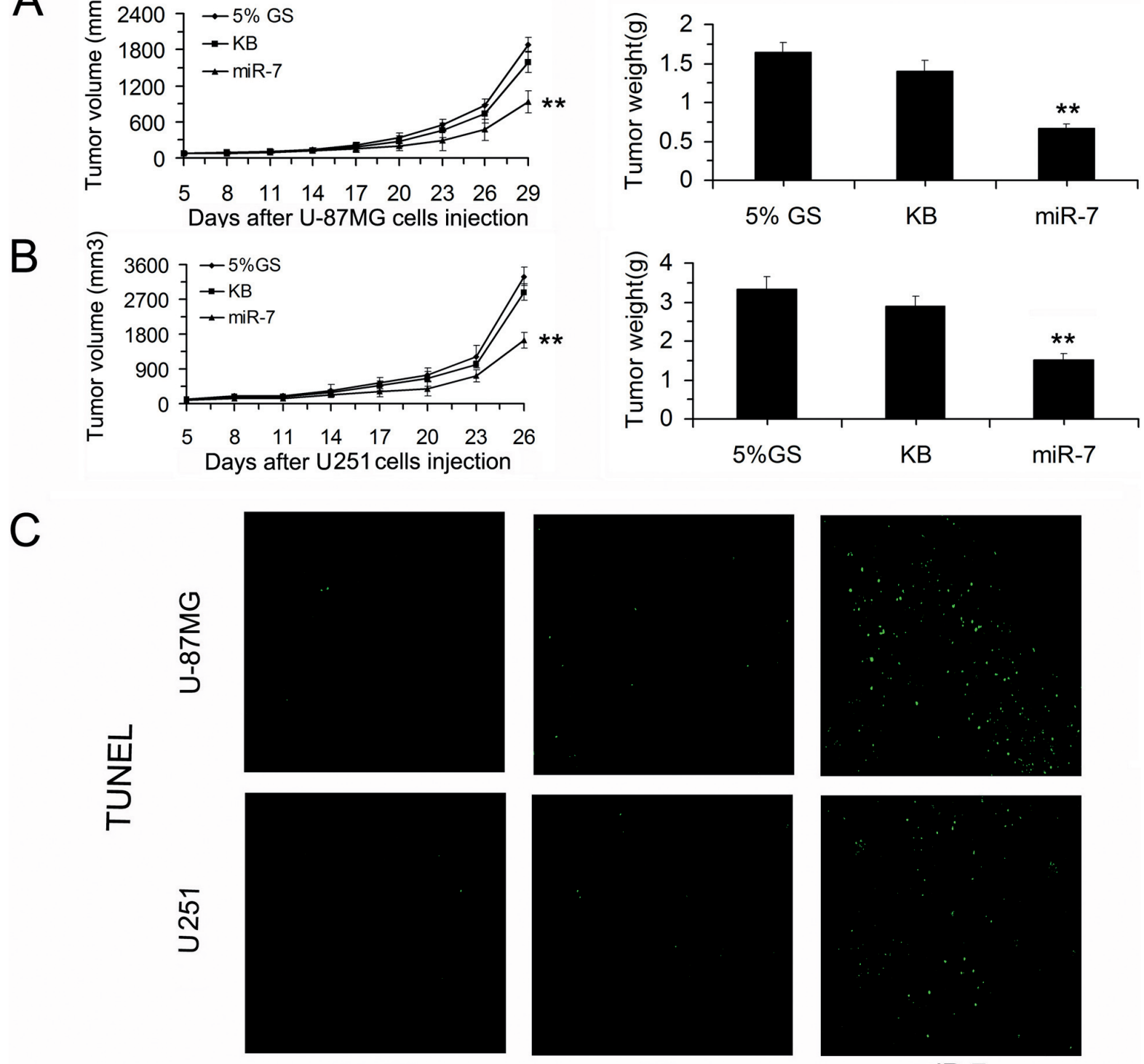

$5 \%$ GS
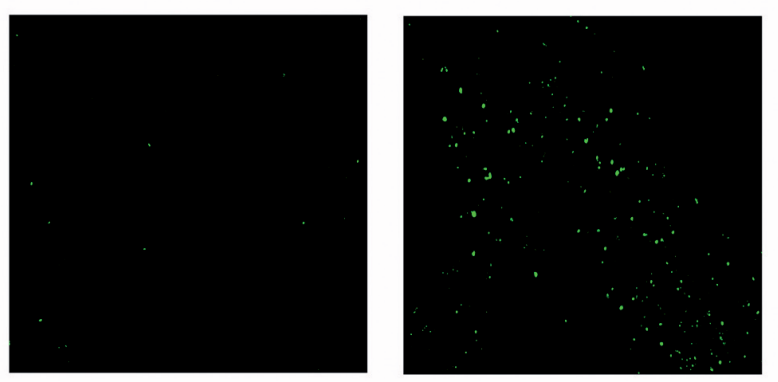

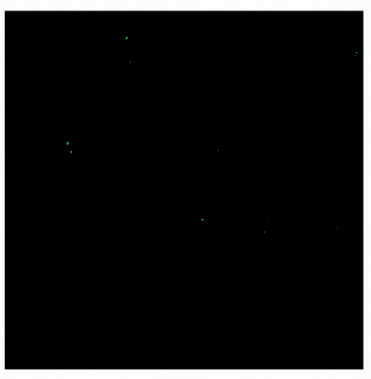

KB

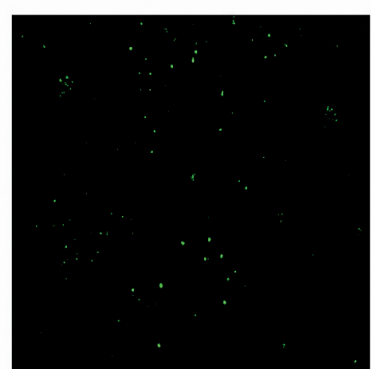

$\operatorname{miR}-7$

Figure 4. miR-7 inhibited glioma subcutaneous xenografts growth and induced cells apoptosis in vivo. Tumor volume and weights in U-87MG (A) or U251 (B) xenografts were significantly reduced ( $n=5$ per group, $P<0.01)$ in miR-7 group compared with control groups. $C$, induction of apoptosis in tumor tissues was indicated by TUNEL assay (original magnification, $\times 200$ ).

tients with cancer. As in our model, we used the U251 human metastatic glioma cells, which had a high metastatic potential and can metastasize to the lung approximately 4 weeks after inoculation [25]. Meanwhile, we found the lymph nodes were swollen. When the tumor xenografts volume reached 3,000 $\mathrm{mm}^{3}$ in our present study, mice were sacrificed and the metastatic nodules on lung surface were counted under a dissecting microscope. A dramatic decrease in the number of tumor metastases was observed in miR-7 treated group compared with others groups (Fig. 6A, C; $P<0.01$ ). H\&E staining of lung and lymph node tissue sections also showed that the miR-7 group had much fewer and smaller metastases than controls (Fig. 6B, $\mathrm{D} ; P<0.01)$.

\section{Discussion}

Cancer cells frequently contain multiple genetic and epigenetic abnormalities. Malignant tumors are generally heterogeneous and caused by the accumulation of genetic alterations in oncogenes and tumor suppressor genes. Despite this complexity, numerous studies indicate that the tumor progression can also be driven by the activity of specific 

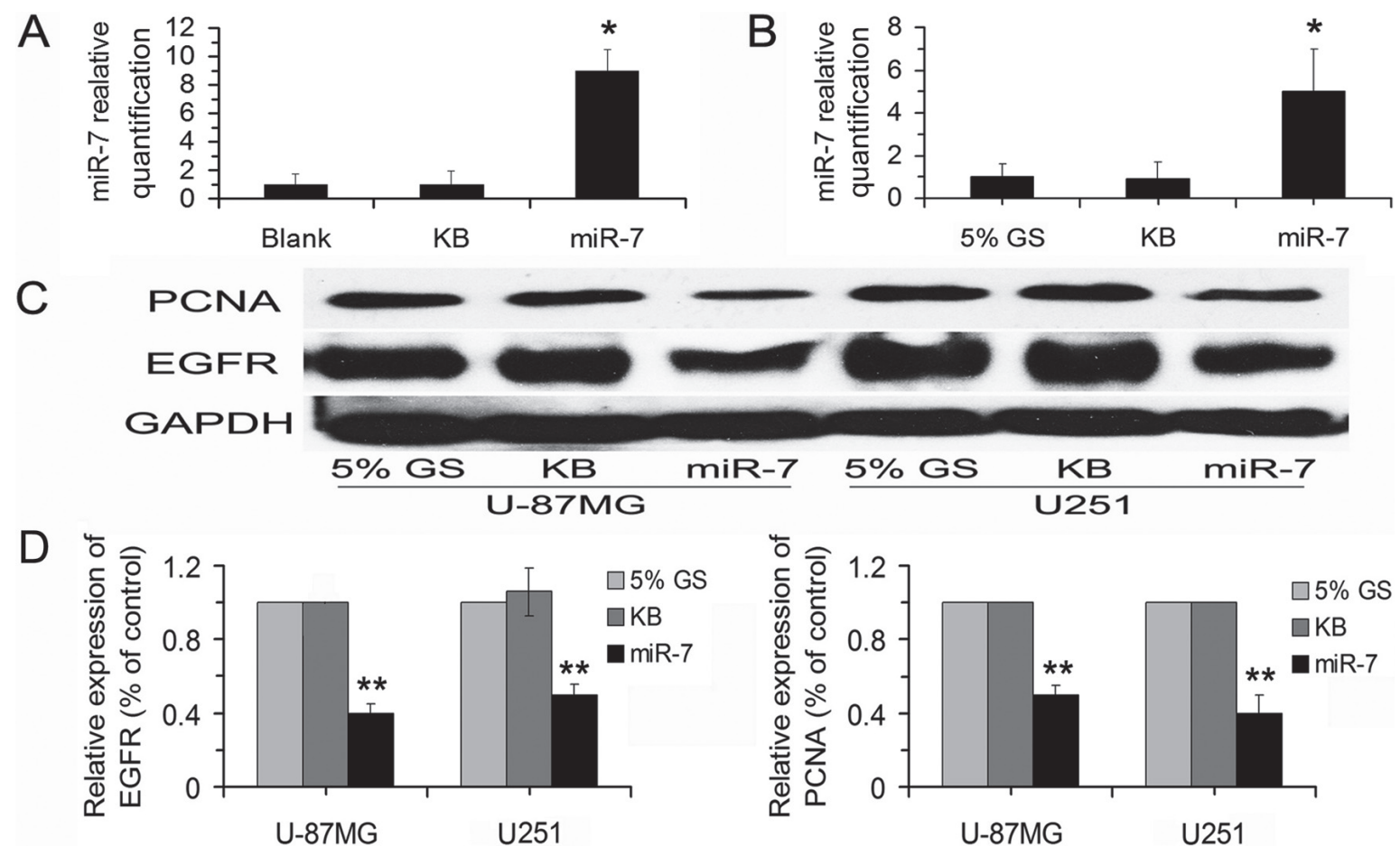

Figure 5. The antitumor effects were caused by miR-7. The miR-7 relative quantification of each group in U251 cells were detected by $\mathrm{qRT}$-PCR in vitro (A) and in vivo (B), miR-7 expression was sharply increased in miR-7 treated group. C and D, expression of EGFR and PCNA were evidently decreased in tumors treated with miR-7 analyzing by western blot $\left(\mathbf{n}=3\right.$; ANOVA; ${ }^{\star *}, P<0.01$ versus controls).

oncogene $[26,27]$. Over the years, based on many molecular genetic and histopathological researches, it is known that GBM is a highly malignant tumor with remarkable genomic heterogeneity, and the EGFR is one of the most deregulated components of human GBM [28, 29]. Consequently, it promoted the development of new anti-EGFR therapeutics such as monoclonal antibodies and tyrosine kinase inhibitors. However, the clinical efficacy of EGFR inhibitor is ultimately limited by the development of acquired drug resistance [30, 31]. Recently, many miRNAs are considered to function as tumor suppressors or oncogenes in human tumors $(10,32$, 33). Factually, it is the promising strategy of using miRNAs for anticancer therapy. Here, we have demonstrated miR-7 as a key tumor suppressor in glioma that functions as a negative regulator of EGFR signaling.

In previous reports, $\mathrm{miR}-7$ has been characterized as tumor suppressors in many human cancer cells and plays important roles in cell proliferation, apoptosis and migration in vitro $[21,34]$. In our present study, we performed mainly further investigation on the therapeutic effect of miR-7 for human glioma in vivo. We used a plasmid-mediated miRNA expression system to express the miR-7 and quantitatively analyzed the anti-tumor effects in vivo. Systematic delivery of miR-7/liposome complex to subcutaneous glioma xenografts induced significant suppression on primary tumor growth (approximately $40 \%$ in tumor volume and tumor weight) and metastatic nodules (60\% suppression ratio in lung metastases and $80 \%$ suppression ratio in lymph node metastases, respectively). These data provided convincing evidences that miR-7 could inhibit glioma xenografts growth and metastasis in vivo and suggested miR-7 might offer a clinically feasible approach for glioma therapy.

Mechanisms of the potent antitumor and anti-metastasis efficacy remain to be fully elucidated. Overexpression of EGFR in human malignancies induces cascades of downstream signaling, such as activation of MAPK/ERK, PI3K/Akt and Jak/Stat, which mediate cell survival, proliferation, apoptosis, invasion, tumor progression and metastasis [13, 33, 34]. MiR-7 targets several proto-oncogenes, including RAF1, IGF1R and PAK1 (18-20). Thus, it is likely that miR7 contributes to tumor progression through multiple genes of multiple pathways. However, our present study demonstrated that miR-7-mediated down-regulation of EGFR led a reduction in cell proliferation, invasion and an enhanced apoptotic rate.

Delivery is still the therapeutic bottleneck in vivo. In this study, we selected the delivery system of DNA-Cationic liposome complexes. The selection of cationic liposome rooted mainly in the parameters of biodegradation, biodistribution, and toxicity [23]. And the validity of DNA-Cationic liposome complexes delivery was supported by previous studies using 

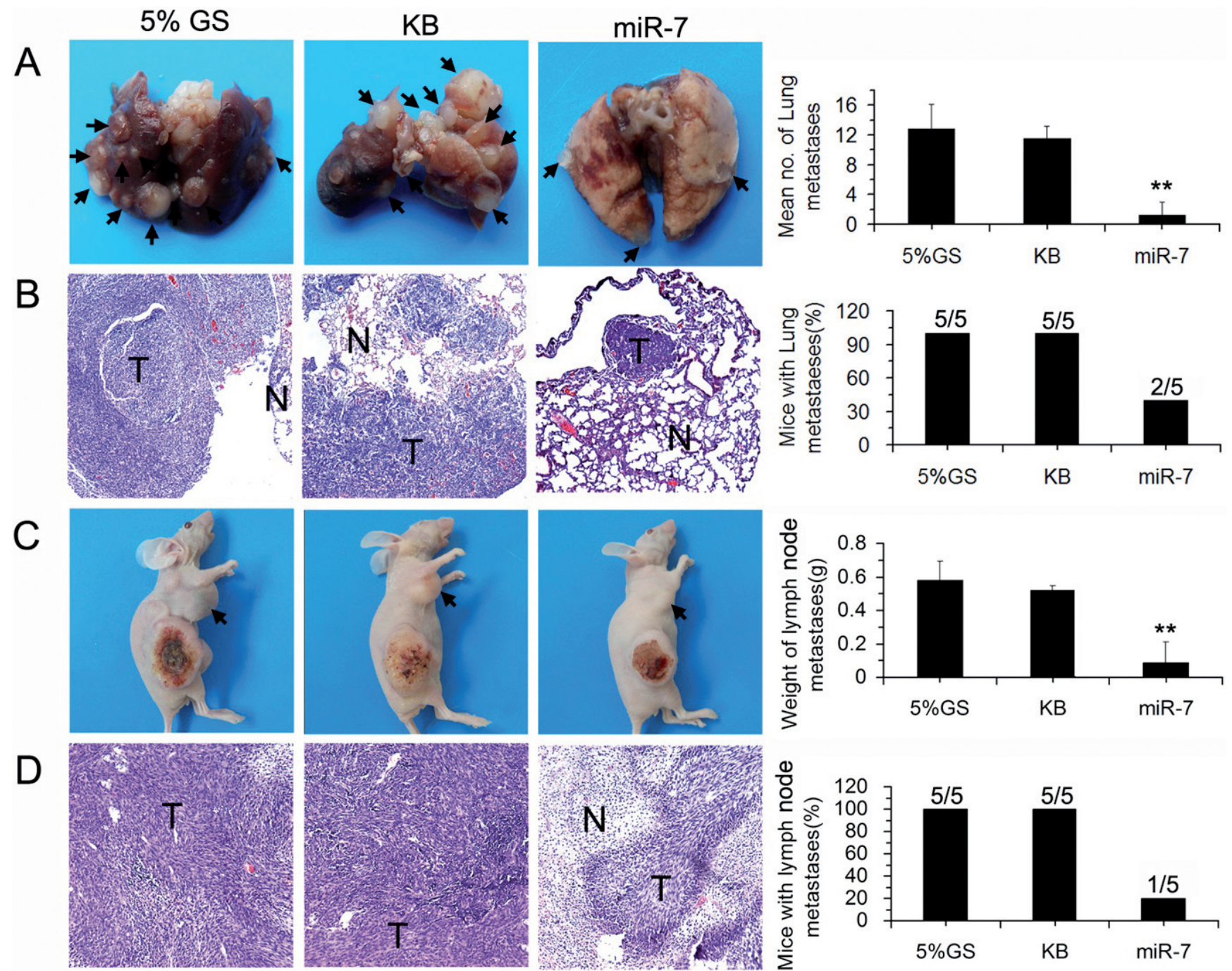

Figure 6. miR-7 suppressed lung and lymph node metastasis in U251 subcutaneous xenograft model. A and C, the number of metastatic nodules on lung surface was calculated from 5 mice per group. Histology was confirmed by H\&E staining (original magnification, $\times 100$ ). B and D, the ratio of lymph node metastasis was calculated from 5 mice per group. Histology was confirmed by H\&E staining (original magnification, $\times 100$ ). Arrows represent the pulmonary metastasis nodules and swollen lymph nodes, respectively; N, Normal tissues; T, Metastatic tumor nodules.

cationic liposome to deliver tumor suppressor genes and inhibit tumor growh [36]. Furthermore, FUS1-Cationic liposome complex had been advanced into phase I clinical trial for treatment of NSCLC [37]. Therefore, the safe and efficient delivery of the cationic liposome vector appeared to be attractive.

In summary, to our knowledge, it is the first demonstration that plasmid-based miR-7 could safely and efficiently suppress glioma xenograft growth and metastases in vivo. The results are encouraging since we regard miR-7 as considerable therapeutic potential suppressor gene for glioma therapy.

Acknowledgements: No potential conflicts of interest were declared. This work is supported by The National Key Basic Research Program (973 Program) of China (2012CB917104).

\section{References}

[1] FURNARI FB, FENTON T, BACHOO RM, MUKASA A, STOMMEL JM et al. Malignant astrocytic glioma: Genetics, biology, and paths to treatment. Genes Dev 2007; 21: 2683-2710. http://dx.doi.org/10.1101/gad.1596707

[2] BARTEL DP MicroRNAs: genomics, biogenesis, mechanism, and function. Cell 2004; 116: 281-297. http://dx.doi. org/10.1016/S0092-8674(04)00045-5

[3] KIM VN, NAM JW Genomics of microRNA. Trends Genet 2006; 22: 165-173. http://dx.doi.org/10.1016/ j.tig.2006.01.003

[4] CARRINGTON JC, AMBROS V Role of microRNAs in plant and animal development. Science 2003; 301: 336-338 http:// dx.doi.org/10.1126/science.1085242 
[5] AMBROS $\mathrm{V}$ The functions of animal microRNAs. Nature 2004; 431: 350-355

[6] CHEN JF, MANDEL EM, THOMSON JM, WU Q, CALLIS TE et al. The role of microRNA-1 and microRNA-133 in skeletal muscle proliferation and differentiation. Nat. Genet 2006; 38: 228-233. http://dx.doi.org/10.1038/ng1725

[7] CHENG AM, Byrom MW, Shelton J, FORD LP Antisense inhibition of human miRNAs and indications for an involvement of miRNA in cell growth and apoptosis. Nucleic Acids Res 2005; 33: 1290-1297. http://dx.doi.org/10.1093/nar/ gki200

[8] KLOOSTERMAN WP, Plasterk RH The diverse functions of microRNAs in animal development and disease. Dev Cell 2006; 11: 441-450. http://dx.doi.org/10.1016/ j.devcel.2006.09.009

[9] CALIN GA, Sevignani C, Dumitru CD, Hyslop T, Noch E et al. Human microRNA genes are frequently located at fragile sites and genomic regions involved in cancers. Proc. Natl. Acad. Sci. USA 2004; 101: 2999-3004. http://dx.doi.org/10.1073/ pnas.0307323101

[10] ZHANG B, PAN X, COBB GP, ANDERSON TA microRNAs as oncogenes and tumor suppressors. Dev Biol. 2007; 302:1-12. http://dx.doi.org/10.1016/j.ydbio.2006.08.028

[11] WIEMER EA The role of microRNAs in cancer: no small matter. Eur J Cancer 2007; 43: 1529-1544. http://dx.doi. org/10.1016/j.ejca.2007.04.002

[12] ESQUELA-KERSCHER A, SLACK FJ Oncomirs-microRNAs with a role in cancer. Nat. Rev. Cancer 2006; 6: 259-269. http://dx.doi.org/10.1038/nrc1840

[13] SALOMON DS, BRANDT R, CIARDIELLO F, NORMANNO $\mathrm{N}$ Epidermal growth factor-related peptides and their receptors in human malignancies. Oncol Hematol 1995; 19: 183-232.

[14] YANO S, KONDO K, YAMAGUCHI M, RICHMOND G, HUTCHISON M et al. Distribution and function of EGFR in human tissue and the effect of EGFR tyrosine kinase inhibition. Anticancer Res 2003; 23: 3639-3650.

[15] LIBERMANN TA, RAZON N, BARTAL AD, YARDEN Y, SCHLESSINGER J et al. Expression of epidermal growth factor receptors in human brain tumors. Cancer Res 1984; 44: 753-760.

[16] HUMPHREY PA, WONG AJ, VOGELSTEIN B, FRIEDMAN HS, WERNER MH et al. Amplification and expression of the epidermal growth factor receptor gene in human glioma xenografts. Cancer Res1988; 48: 2231-2238.

[17] SHINOJIMA N, TADA K, SHIRAISHI S, KAMIRYO T, KOCHI $\mathrm{M}$ et al. Prognostic value of epidermal growth factor receptor in patients with glioblastoma multiforme. Cancer Res 2003; 63: 6962-6970.

[18] JIANG L, LIU X, CHEN Z, JIN Y, HEIDBREDER CE et al. MicroRNA-7 targets IGF1R (insulin-like growth factor 1 receptor) in tongue squamous cell carcinoma cells. Biochem J 2010; 432: 199-205. http://dx.doi.org/10.1042/ B)20100859

[19] REDDY SD, OHSHIRO K, RAYALA SK, KUMAR R MicroRNA-7, a homeobox D10 target, inhibits p21-activated kinase 1 and regulates its functions. Cancer Res 2008; 68:
8195-8200. http://dx.doi.org/10.1158/0008-5472.CAN-08$\underline{2103}$

[20] WEBSTER RJ, GILES KM, PRICE KJ, ZHANG PM, MATTICK JS et al. Regulation of Epidermal Growth Factor Receptor Signaling in Human Cancer Cells by MicroRNA-7. J Biol Chem. 2009; 284: 5731-5741. http://dx.doi.org/10.1074/ jbc.M804280200

[21] KEFAS B, GODLEWSKI J, COMEAU L, LI Y, ABOUNADER $\mathrm{R}$ et al. microRNA-7 Inhibits the Epidermal Growth Factor Receptor and the Akt Pathway and Is Down-regulated in Glioblastoma. Cancer Res 2008; 68: 3566-3572. http://dx.doi. org/10.1158/0008-5472.CAN-07-6639

[22] GAUR A, JEWELL DA, LIANG Y, RIDZON D, MOORE JH et al. Characterization of microRNA expression levels and their biological correlates in human cancer cell lines. Cancer Res 2007; 67: 2456-2468. http://dx.doi.org/10.1158/0008-5472. CAN-06-2698

[23] TEMPLETON NS, LASIC DD, FREDERIK PM, STREY HH, ROBERTS DD et al. Improved DNA: liposome complexes for increased systemic delivery and gene expression. Nat. Biotechnol 1997; 15: 647-652. http://dx.doi.org/10.1038/ $\underline{\text { nbt0797-647 }}$

[24] DAI L, WANG W, ZHANG S, JIANG Q, WANG R, et al. Vector-based miR-15a/16-1 plasmid inhibits colon cancer growth in vivo. Cell Biol Int. 2012; 36: 765-770. http://dx.doi. org/10.1042/CBI20110404

[25] LU ZJ, LIU SY, YAO YQ, ZHOU YJ, ZHANG S et al. The effect of miR-7 on biological behavior and global protein expression in glioma cell lines. Electrophoresis 2011; 32: 3612-3620. http://dx.doi.org/10.1002/elps.201100230

[26] WEINSTEIN IB Addiction to oncogenes-the Achilles Heal of Cancer. Science 2002; 297: 63-64. http://dx.doi.org/10.1126/ science. 1073096

[27] WEINSTEIN IB, JOE A Oncogene Addiction. Cancer Res 2008; 68: 3077-3080. http://dx.doi.org/10.1158/0008-5472. CAN-07-3293

[28] THE CANCER GENOME ATLAS RESEARCH NETWORK Comprehensive genomic characterization defines human glioblastoma genes and core pathways. Nature 2008; 455: 1061-1068. http://dx.doi.org/10.1038/nature07385

[29] PARSONS DW, JONES S, ZHANG X, LIN JC, LEARY RJ et al. An Integrated Genomic Analysis of Human Glioblastoma Multiforme. Science 2008; 321: 1807-1812. http://dx.doi. org/10.1126/science. 1164382

[30] RITTER CA, ARTEAGA CL The epidermal growth factor receptor-tyrosine kinase: a promising therapeutic target in solid tumors. Semin Oncol 2003; 30: 3-11. http://dx.doi. org/10.1053/sonc.2003.50027

[31] BIANCO R, TROIANI T, TORTORA G, CIARDIELLO F Intrinsic and acquired resistance to EGFR inhibitors in human cancer therapy. Endocr Relat Cancer 2005; 12: S159-S171. http://dx.doi.org/10.1677/erc.1.00999

[32] RUAN K, FANG X, OUYANG G MicroRNAs: Novel regulators in the hallmarks of human cancer. Cancer Lett 2009; 285: 116-126. http://dx.doi.org/10.1016/j.canlet.2009.04.031

[33] CROCE MC Oncogenes and Cancer. N Engl J Med 2008; 358: 502-511. http://dx.doi.org/10.1056/NEJMra072367 
[34] WELLS A EGF receptor Int J Biochem Cell Biol 1999; 31: 637-643. http://dx.doi.org/10.1016/S1357-2725(99)00015$\underline{1}$

[35] LURJE G, LENZ HJ EGFR Signaling and Drug Discovery. Oncology 2009; 77: 400-410. http://dx.doi.org/10.1159/ $\underline{000279388}$
[36] LU C, STEWART DJ, JI L Systemic gene therapy with tumor suppressor FUS1-nanoparticles for recurrent/metastatic lung cancer. J Clin Oncol 2010; 28: 7582.

[37] LU C, STEWART DJ, JI L, RAMESH R, JAYACHANDRAN G et al. A phase I trial of intravenous therapy with tumor suppressor FUS1-nanoparticles. J Clin Oncol 2009; 27: e19065. 